\title{
Counting peaks and valleys in $k$-colored Motzkin paths
}

\author{
A. Sapounakis and P. Tsikouras \\ Department of Informatics \\ University of Piraeus, Piraeus, GREECE \\ arissap@unipi.gr, pgtsik@unipi.gr
}

Submitted: Oct 6, 2004; Accepted: Mar 10, 2005; Published Mar 18, 2005

Mathematics Subject Classifications: 05A15, 05A19

\begin{abstract}
This paper deals with the enumeration of $k$-colored Motzkin paths with a fixed number of (left and right) peaks and valleys. Further enumeration results are obtained when peaks and valleys are counted at low and high level. Many well-known results for Dyck paths are obtained as special cases.
\end{abstract}

\section{Introduction}

A wide range of articles dealing with Dyck and Motzkin paths and related topics appears frequently in the literature (e.g., $[1,7,9,12,13,14,15,20]$ ). More generally, $k$-colored Motzkin paths $[2,17]$ which have horizontal steps colored by means of $k$ colors, are of particular interest and have important applications (e.g., [3, 4, 8, 17] for $k=2$ and [11, 17] for $k=3)$.

In this paper, several enumeration results for the set $\mathcal{M}$ of $k$-colored Motzkin paths, according to various parameters are established, with the aid of generating functions. Most of these results are known for $k=0$ (i.e., for Dyck paths), while they are new even for $k=1$ (i.e., for Motzkin paths).

In section 2, some basic definitions and notations referring to the set $\mathcal{M}$ and various parameters of it are given.

In section 3, using some simple bijections, several parameters of $\mathcal{M}$ are categorized into classes, the elements of which are equidistributed. Then, by picking a parameter from each class (e.g. the number of left peaks, right valleys, double rises and peaks) the generating function of $\mathcal{M}$ is found according to length, number of rises and this parameter, giving several enumeration results.

In section 4 (resp. section 5), parameters related to peaks and valleys at low (resp. high) level are considered. Several well-known results on Dyck paths are generalized to $k$-colored Motzkin paths. For example, it is shown that the parameters "number of high peaks" and "number of valleys" are equidistributed in $\mathcal{M}$. This result is also shown by constructing a bijection on the set $\mathcal{M}$. 


\section{Preliminaries}

A $k$-colored Motzkin path of length $n$ is a lattice path of $\mathbb{N}^{2}$ running from $(0,0)$ to $(n, 0)$, that never goes below the $x$-axis and whose allowed steps are the up diagonal step $(1,1)$, the down diagonal step $(1,-1)$ and the colored horizontal step $(1,0)$ which is labeled by means of $k$ colors, $k \in \mathbb{N}$. These steps are called rise, fall and level step respectively.

In the cases $k=0,1$ we obtain the well-known Dyck and Motzkin paths, enumerated by the Catalan numbers $C_{\frac{n}{2}}$, for $n$ even (A000108) and the Motzkin numbers $M_{n}$ (A001006) respectively, [18]. On the other hand the number of 2-colored (resp. 3-colored) Motzkin paths of length $n$ is equal to $C_{n+1}$ (resp. $\left.\sum_{m=0}^{n}\left(\begin{array}{c}n \\ m\end{array}\right) C_{m+1}\right),[8,17]$.

In this work we restrict ourselves to the case where $k \neq 0$, though all the results of this paper remain true for $k=0$.

It is clear that each $k$-colored Motzkin path is coded by a word $u=u_{1} u_{2} \cdots u_{n} \in$ $\left\{a, \bar{a}, \beta_{1}, \beta_{2}, \ldots, \beta_{k}\right\}^{*}$, called $k$-colored Motzkin word, so that every rise (resp. fall) corresponds to the letter $a$ (resp. $\bar{a}$ ) and every colored level step corresponds to a certain $\beta_{i}$, $i \in[k]=\{1,2, \ldots, k\}$; see Fig. 1 .

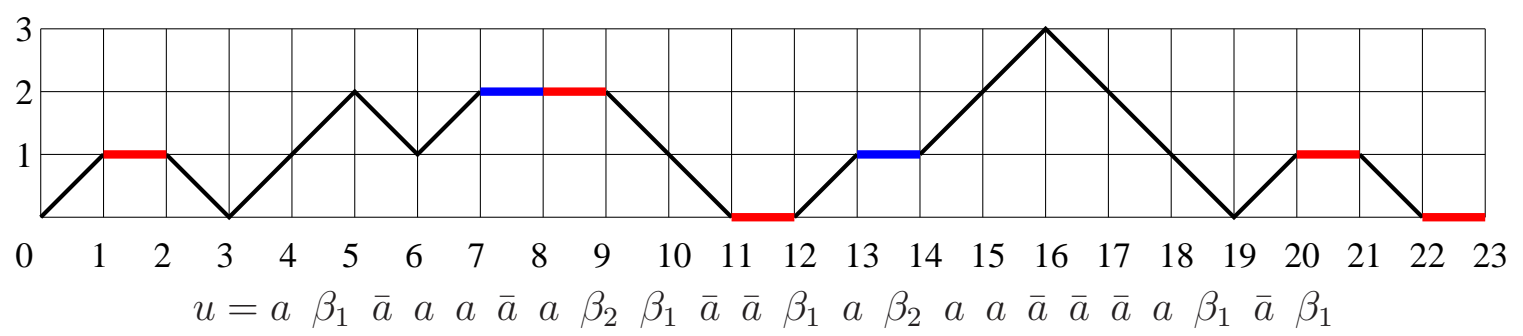

Figure 1: A 2-colored Motzkin path and its corresponding word

Throughout this paper we denote by $\mathcal{M}$ the set of all $k$-colored Motzkin words (or equivalently $k$-colored Motzkin paths). Its subset consisting of all the words in $\{a, \bar{a}\}^{*}$ is the set $\mathcal{D}$ of Dyck words. Furthermore the subset of $\mathcal{M}$ which contains the words $u$ of length $l(u)=n$ with $r(u)=r$ rises, where $0 \leq r \leq\left[\frac{n}{2}\right]$ is denoted by $\mathcal{M}_{n, r}$. In particular we write $\mathcal{D}_{r}=\mathcal{M}_{2 r, r}$ for the set of Dyck words of length $2 r$.

It is clear that each non-empty word $u=u_{1} u_{2} \cdots u_{n} \in \mathcal{M}$ can be uniquely written in either of the forms $u=\beta_{\nu} z$, or $u=a w \bar{a} z$, where $w, z \in \mathcal{M}$ and $\nu \in[k]$.

It follows that the sets

and

$$
\mathcal{A}=\left\{u \in \mathcal{M}: u_{1}=a\right\}
$$

$$
\mathcal{B}=\left\{u \in \mathcal{M}: u_{1}=\beta_{\nu}, \nu \in[k]\right\} \cup\{\epsilon\}
$$

where $\epsilon$ is the empty word, form a partition of $\mathcal{M}$.

For a parameter $q$ defined on $\mathcal{M}$ we will denote by $F_{q}$ the generating function of $\mathcal{M}$ according to the parameters $l, r$ and $q$ i.e.,

$$
F_{q}(x, y, t)=\sum_{u \in \mathcal{M}} x^{l(u)} y^{r(u)} t^{q(u)}
$$


Similarly, we denote by $A_{q}, B_{q}$ the generating functions of $\mathcal{A}, \mathcal{B}$ respectively, according to the parameters $l, r$ and $q$.

If $q(u)=0$ for all $u \in \mathcal{M}$, then by $F(x, y), A(x, y)$, and $B(x, y)$ we denote the generating functions of the sets $\mathcal{M}, \mathcal{A}$ and $\mathcal{B}$, respectively, according to the parameters $l$ and $r$.

Using the partition $\{\mathcal{A}, \mathcal{B}\}$ of $\mathcal{M}$, we obtain at once the following equation ([17], Proposition 3.1).

$$
x^{2} y F^{2}(x, y)+(k x-1) F(x, y)+1=0
$$

and the coefficients of the powers $F^{s}(x, y), s \in \mathbb{N}^{*}$ are given by the formula:

$$
\left[x^{n} y^{r}\right] F^{s}=\frac{s}{n+s}\left(\begin{array}{c}
n+s \\
s+r, r, n-2 r
\end{array}\right) k^{n-2 r} .
$$

Two parameters $q_{1}, q_{2}$ of $\mathcal{M}$ are called equidistributed if

$$
\left|\left\{u \in \mathcal{M}_{n, r}: q_{1}(u)=\mu\right\}\right|=\left|\left\{u \in \mathcal{M}_{n, r}: q_{2}(u)=\mu\right\}\right|
$$

for every $n, r, \mu \in \mathbb{N}$.

A point of a $k$-colored Motzkin path is called peak (resp. valley) if it is preceded by a rise (resp. fall) and followed by a fall (resp. rise). A left peak (resp. left valley) is preceded by a rise (resp. fall) and followed by either a level step or a fall (resp. rise). Obviously, a point of a $k$-colored Motzkin path is a peak (resp. valley) if and only if it is both left and right peak (resp. valley). The right peak and the right valley are defined in an analogous way. A peak or a valley is at height $k$ if its $y$-coordinate is $k$.

A double rise (resp. double fall) occurs at a point preceded as well as followed by a rise (resp. fall). A left double rise (resp. left double fall) occurs at a point preceded by a rise (resp. fall) and followed by either a level step or a rise (resp. fall). The right double rise and the right double fall are defined in an analogous way; see Fig. 2.

\section{$3 \quad$ Enumeration according to various parameters}

In this section we present several enumeration results, using the generating functions of $k$ colored Motzkin paths according to length, number of rises and various other parameters.

We will study the parameters of $\mathcal{M}: l p, r p, p, l v, r v, v, l d r, r d r, d r, l d f, r d f$ and $d f$ defined by the number of left peaks, right peaks, peaks, left valleys, right valleys, valleys, left double rises, right double rises, double rises, left double falls, right double falls and double falls respectively.

It is easy to see by considerations of symmetry that if $\left\{q_{1}, q_{2}\right\}$ is anyone of the pairs $\{l p, r p\},\{l v, r v\},\{l d r, r d f\},\{r d r, l d f\}$ and $\{d r, d f\}$, then the parameters $q_{1}, q_{2}$ are equidistributed.

Furthermore, we will show that if $\left\{q_{1}, q_{2}\right\}$ is anyone of the pairs $\{d r, v\},\{l d r, l v\}$ and $\{r d r, r v\}$ then the parameters $q_{1}, q_{2}$ are equidistributed. 


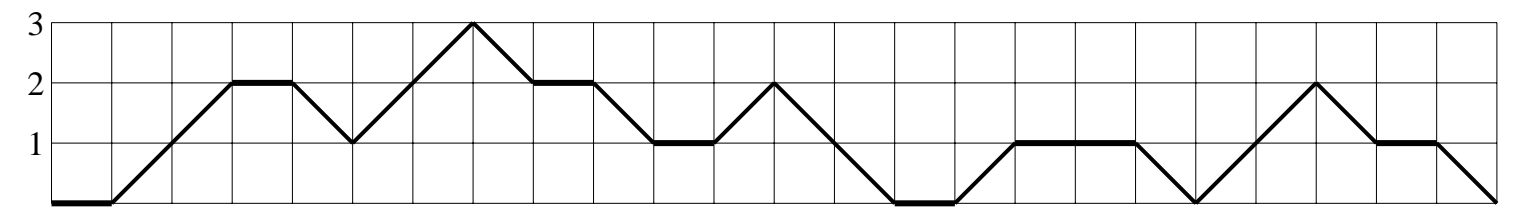

$\begin{array}{lllllllllllllllllllllllll}0 & 1 & 2 & 3 & 4 & 5 & 6 & 7 & 8 & 9 & 10 & 11 & 12 & 13 & 14 & 15 & 16 & 17 & 18 & 19 & 20 & 21 & 22 & 23 & 24\end{array}$

peaks : $7,12,21$

left peaks : $3,7,12,16,21$

left valleys : $5,8,10,14,19,22$

double rises : $2,6,20$

left double rises : 2, 3, 6, 16, 20 valleys : 5,19

right peaks : 4, 7, 9, 12, 18, 21, 23

right valleys : $1,5,11,15,19$

double falls : 13

right double rises : $1,2,6,11,15,20$

right double falls : 4, 9, 13, 18, 23

Figure 2: Various kinds of points of a Motzkin path;

(each point is coded by its $x$-coordinate).

To see this, we consider an involution $\theta$ of $\mathcal{M}$ which is defined as a natural extension of the involution of $\mathcal{D}$ used in [6] in order to show that the parameters $d r$ and $v$ are equidistributed in $\mathcal{D}$.

The definition of $\theta$ is given recursively: If $u=\epsilon$ we set $\theta(\epsilon)=\epsilon$. Next, for $n \in \mathbb{N}^{*}$ and assuming that $\theta(z)$ has been defined for each $z \in \mathcal{M}$ with $l(z)<n$, we set

$$
\theta(u)= \begin{cases}\beta_{\nu} \theta(z), & \text { if } u=\beta_{\nu} z, \nu \in[k], z \in \mathcal{M} \\ a \theta(z) \bar{a} \theta(w), & \text { if } u=a w \bar{a} z, w, z \in \mathcal{M}\end{cases}
$$

It is easy to check by induction that $\theta$ is an involution of $\mathcal{M}$ such that $l(\theta(u))=l(u)$, $r(\theta(u))=r(u)$ for each $u \in \mathcal{M}$ and $\theta(\mathcal{A})=\mathcal{A}, \theta(\mathcal{B})=\mathcal{B}$.

Furthermore, we show by induction that $d r(\theta(u))=v(u)$, for each $u \in \mathcal{M}$.

Indeed, if $u=\beta_{\nu} z$ for some $\nu \in[k]$ and $z \in \mathcal{M}$, then

$$
d r(\theta(u))=d r(\theta(z))=v(z)=v(u) .
$$

If on the other hand $u=a w \bar{a} z$ for some $w, z \in \mathcal{M}$, then

$$
\begin{aligned}
d r(\theta(u)) & = \begin{cases}d r(\theta(z))+d r(\theta(w))+1, & \text { if } \theta(z) \in \mathcal{A} \\
d r(\theta(z))+d r(\theta(w)), & \text { if } \theta(z) \in \mathcal{B}\end{cases} \\
& = \begin{cases}v(z)+v(w)+1, & \text { if } z \in \mathcal{A} \\
v(z)+v(w), & \text { if } z \in \mathcal{B}\end{cases} \\
& =v(u) .
\end{aligned}
$$

In the same way it can be shown that $l d r(\theta(u))=l v(u)$ and $r d r(\theta(u))=r v(u)$, for each $u \in \mathcal{M}$. From the previous discussion we deduce the next result. 
Proposition 3.1 The following parameters are equidistributed in $\mathcal{M}$ :

i. $l p$ and $r p$.

ii. $d r$, df and $v$.

iii. $l d r, r d r, l d f, r d f, l v$ and $r v$.

In view of the previous result, it is enough to investigate the parameters $l p, d r, r v$ and $p$. For this we use the following result.

Lemma 3.2 The generating function $F(x, y, s, t)$ of $\mathcal{M}$ according to the parameters $l, r, p$ and $q$, where $q(u)$ is the number of occurences of $a \beta_{\nu}, \nu \in[k]$, in a word $u \in \mathcal{M}$, satisfies the following equation:

$$
F=1+k x F+x^{2} y(s+k x t F+F-1-k x F) F .
$$

Proof: Each non-empty word $u=u_{1} u_{2} \cdots u_{n} \in \mathcal{M}$ can be uniquely written in one of the forms $u=\beta_{\nu} w_{1}, u=a \bar{a} w_{1}, u=a \beta_{\nu} w_{1} \bar{a} w_{2}$ or $u=a a w_{1} \bar{a} w_{2} \bar{a} w_{3}$, where $\nu \in[k]$ and $w_{1}, w_{2}, w_{3} \in \mathcal{M}$. So, we obtain that

$$
\begin{aligned}
F & =1+k x F+x^{2} y s F+k x^{3} y t F^{2}+x^{4} y^{2} F^{3} \\
& =1+k x F+x^{2} y\left(s+k x t F+x^{2} y F^{2}\right) F \\
& =1+k x F+x^{2} y(s+k x t F+F-1-k x F) F .
\end{aligned}
$$

For the proof of the next result we need the well-known Vandermonde convolution formula $([10],(3.1))$

$$
\sum_{\rho=0}^{\mu}\left(\begin{array}{c}
r \\
\mu-\rho
\end{array}\right)\left(\begin{array}{l}
\nu \\
\rho
\end{array}\right)=\left(\begin{array}{c}
r+\nu \\
\mu
\end{array}\right)
$$

as well as the formula

$$
\sum_{\nu=(-\mu)^{+}}^{r}(-1)^{r+\nu}\left(\begin{array}{l}
r \\
\nu
\end{array}\right)\left(\begin{array}{c}
m+\mu+\nu \\
\mu+\nu
\end{array}\right)=\left(\begin{array}{c}
m+\mu \\
r+\mu
\end{array}\right)
$$

(where $(-\mu)^{+}=\max (-\mu, 0)$ and $\left.-\mu \leq r.\right)$, for $\mu \geq 0([10],(3.48)$ ).

Proposition 3.3 The generating function $F_{l p}$ satisfies the following equation:

$$
\left(k x^{3} y(t-1)+x^{2} y\right) F_{l p}^{2}(x, y, t)+\left(x^{2} y(t-1)+k x-1\right) F_{l p}(x, y, t)+1=0 .
$$

Furthermore the coefficients of the powers $F_{l p}^{s}(x, y, t), s \in \mathbb{N}^{*}$ are given by the formula

$$
\left[x^{n} y^{r} t^{\gamma}\right] F_{l p}^{s}=\frac{s}{r}\left(\begin{array}{c}
n-r+s \\
n-2 r
\end{array}\right)\left(\begin{array}{l}
r \\
\gamma
\end{array}\right)\left(\begin{array}{c}
n-r+s-1 \\
\gamma-1
\end{array}\right) k^{n-2 r}
$$

for every $1 \leq \gamma \leq r$. 
Proof: Clearly, since $F_{l p}(x, y, t)=F(x, y, t, t)$, equation (5) follows at once from Lemma 3.2.

We now come to find the coefficients of the powers $F_{l p}^{s}(x, y, t)$, for $s \geq 1$. For this, we set $\phi=x(t-1)$ and $H(x)=x F_{l p}(x, y, t)$ where $y$ and $t$ are considered as parameters. Using the equation obtained above, we have that

$$
x\left((k y \phi+y) H^{2}(x)+(\phi y+k) H(x)+1\right)=H(x) .
$$

If we set $P(\lambda)=(k \phi+1) y \lambda^{2}+(\phi y+k) \lambda+1$ then $H(x)=x P(H(x))$ and $P(0)=1$. Using the Lagrange inversion formula [19], we obtain that

$$
\left[x^{\sigma}\right] H^{s}=\frac{1}{\sigma}\left[\lambda^{\sigma-1}\right]\left(s \lambda^{s-1}(P(\lambda))^{\sigma}\right) .
$$

Furthermore, we have

$$
\begin{aligned}
\frac{s}{\sigma} \lambda^{s-1}(P(\lambda))^{\sigma} & =\frac{s}{\sigma} \lambda^{s-1} \sum_{i=0}^{\sigma}\left(\begin{array}{l}
\sigma \\
i
\end{array}\right) \lambda^{i}((k \phi+1) y \lambda+(\phi y+k))^{i} \\
& =\frac{s}{\sigma} \sum_{i=0}^{\sigma} \sum_{\xi=0}^{i}\left(\begin{array}{c}
\sigma \\
i
\end{array}\right)\left(\begin{array}{c}
i \\
\xi
\end{array}\right)(\phi y+k)^{i-\xi} y^{\xi}(k \phi+1)^{\xi} \lambda^{\xi+i+s-1} \\
& =\frac{s}{\sigma} \sum_{m=0}^{2 \sigma} \sum_{\xi=(m-\sigma)^{+}}^{\left[\frac{m}{2}\right]}\left(\begin{array}{c}
\sigma \\
m-\xi
\end{array}\right)\left(\begin{array}{c}
m-\xi \\
\xi
\end{array}\right)(\phi y+k)^{m-2 \xi} y^{\xi}(k \phi+1)^{\xi} \lambda^{m+s-1} .
\end{aligned}
$$

If we set $m=\sigma-s$ we obtain that

$$
\left[x^{\sigma}\right] H^{s}=\frac{s}{\sigma} \sum_{\xi=0}^{\left[\frac{\sigma-s}{2}\right]}\left(\begin{array}{c}
\sigma \\
\sigma-s-\xi
\end{array}\right)\left(\begin{array}{c}
\sigma-s-\xi \\
\xi
\end{array}\right)(\phi y+k)^{\sigma-s-2 \xi} y^{\xi}(k \phi+1)^{\xi}
$$

for every $\sigma \geq s$.

Applying the previous equality for $\sigma+s$ instead of $\sigma$, after some simple manipulations we deduce that

$$
\begin{aligned}
& F_{l p}^{s}(x, y, t)=\sum_{\sigma=0}^{\infty} \frac{s}{\sigma+s} \sum_{\xi=0}^{\left[\frac{\sigma}{2}\right]}\left(\begin{array}{c}
\sigma+s \\
\sigma-\xi
\end{array}\right)\left(\begin{array}{c}
\sigma-\xi \\
\xi
\end{array}\right)(\phi y+k)^{\sigma-2 \xi} y^{\xi}(\phi k+1)^{\xi} x^{\sigma} \\
& =\sum_{\sigma=0}^{\infty} \sum_{\xi=0}^{\left[\frac{\sigma}{2}\right]} \sum_{j=0}^{\sigma-2 \xi} \sum_{\rho=0}^{\xi} \sum_{\gamma=0}^{\rho+j}(-1)^{\rho+j-\gamma} \frac{s}{\sigma+s}\left(\begin{array}{c}
\rho+j \\
\gamma
\end{array}\right)\left(\begin{array}{c}
s+\sigma \\
s+\xi, j, \sigma-2 \xi-j, \rho, \xi-\rho
\end{array}\right) . \\
& \cdot k^{\sigma-2 \xi-j+\rho} y^{j+\xi} t^{\gamma} x^{j+\rho+\sigma} \\
& =\sum_{n=0}^{\infty} \sum_{r=0}^{\left[\frac{n}{2}\right]} \sum_{\gamma=0}^{r} \sum_{\nu=0}^{r-\gamma} \sum_{\rho=0}^{\min \{r-\nu, n-2 r\}}\left(\begin{array}{c}
-1)^{r-\gamma-\nu} \frac{s}{n-r+\nu+s}\left(\begin{array}{c}
r-\nu \\
\gamma
\end{array}\right) . \\
n-r+\nu+s \\
\nu+\rho+s, r-\rho-\nu, n-2 r-\rho, \rho, \nu
\end{array}\right) k^{n-2 r} x^{n} y^{r} t^{\gamma} .
\end{aligned}
$$


It follows that

$$
\begin{aligned}
{\left[x^{n} y^{r} t^{\gamma}\right] F_{l p}^{s} } & =\frac{s k^{n-2 r}}{(n-2 r) !(r+s) !} \sum_{\nu=0}^{r-\gamma}\left(\begin{array}{c}
r-\nu \\
\gamma
\end{array}\right) \frac{(n-r+\nu+s-1) !}{\nu !} \sum_{\rho=0}^{r-\nu}\left(\begin{array}{c}
r+s \\
r-\nu-\rho
\end{array}\right)\left(\begin{array}{c}
n-2 r \\
\rho
\end{array}\right) \\
& =\frac{s}{r}\left(\begin{array}{c}
n-r+s \\
n-2 r
\end{array}\right)\left(\begin{array}{l}
r \\
\gamma
\end{array}\right) k^{n-2 r} \sum_{\nu=0}^{r-\gamma}(-1)^{r-\gamma-\nu}\left(\begin{array}{c}
r-\gamma \\
\nu
\end{array}\right)\left(\begin{array}{c}
n-r+\nu+s-1 \\
r-1
\end{array}\right) \\
& =\frac{s}{r}\left(\begin{array}{c}
n-r+s \\
n-2 r
\end{array}\right)\left(\begin{array}{l}
r \\
\gamma
\end{array}\right)\left(\begin{array}{c}
n-r+s-1 \\
\gamma-1
\end{array}\right) k^{n-2 r}
\end{aligned}
$$

for every $1 \leq \gamma \leq r$.

Remark Notice that if $\gamma=0$ then $r=0$ too. In this case we have $\left[x^{n} y^{0} t^{0}\right] F_{l p}^{s}=\left(\begin{array}{c}n+s-1 \\ s-1\end{array}\right) k^{n}$.

As we have already pointed out, it is enough to deal now with the parameters $r v, d r$ and $p$. Though these parameters can be studied independently using a method analogous to that of the proof of Proposition 3.3, we will investigate them in relation to the parameter $l p$.

We can easily show that $r v$ is expressed in terms of $l p$ as follows:

$$
r v(u)= \begin{cases}\operatorname{lp}(u)-1, & \text { if } u \in \mathcal{A} ; \\ l p(u), & \text { if } u \in \mathcal{B}\end{cases}
$$

Using relation (7) we derive the formula

$$
F_{r v}(x, y, t)=1-t^{-1}+\left(t^{-1}+k x\left(1-t^{-1}\right)\right) F_{l p}(x, y, t)
$$

Furthermore we obtain the following result.

Proposition 3.4 The number of all $u \in \mathcal{M}_{n, r}$ with $\gamma$ right valleys is equal to

$$
\left[x^{n} y^{r} t^{\gamma}\right] F_{r v}=\frac{1}{n-r}\left(\begin{array}{c}
n-r \\
r
\end{array}\right)\left(\begin{array}{l}
r \\
\gamma
\end{array}\right)\left(\begin{array}{c}
n-r \\
\gamma+1
\end{array}\right) k^{n-2 r}
$$

where $0 \leq \gamma \leq r$

Proof: Let $a_{n, r, \gamma}=\frac{1}{r}\left(\begin{array}{c}n-r+1 \\ n-2 r\end{array}\right)\left(\begin{array}{c}r \\ \gamma\end{array}\right)\left(\begin{array}{c}n-r \\ \gamma-1\end{array}\right)$, where $1 \leq \gamma \leq r$.

From proposition 3.3 and relation (8) it follows that

$$
\begin{aligned}
F_{r v}(x, y, t)= & 1-t^{-1}+\left(t^{-1}+k x\left(1-t^{-1}\right)\right)\left(\frac{1}{1-k x}+\sum_{n=2}^{\infty} \sum_{r=1}^{\left[\frac{n}{2}\right]} \sum_{\gamma=1}^{r} a_{n, r, \gamma} k^{n-2 r} x^{n} y^{r} t^{\gamma}\right) \\
= & \frac{1}{1-k x}+\sum_{n=2}^{\infty} \sum_{r=1}^{\left[\frac{n}{2}\right]} \sum_{\gamma=0}^{r-1} a_{n, r, \gamma+1} k^{n-2 r} x^{n} y^{r} t^{\gamma}+ \\
& +\sum_{n=3}^{\infty} \sum_{r=1}^{\left[\frac{n-1}{2}\right]} \sum_{\gamma=1}^{r} a_{n-1, r, \gamma} k^{n-2 r} x^{n} y^{r} t^{\gamma}-\sum_{n=3}^{\infty} \sum_{r=1}^{\left[\frac{n-1}{2}\right]} \sum_{\gamma=0}^{r-1} a_{n-1, r, \gamma+1} k^{n-2 r} x^{n} y^{r} t^{\gamma}
\end{aligned}
$$


We show the desired formula when $1 \leq \gamma \leq r-1$ and $1 \leq r \leq\left[\frac{n-1}{2}\right]$. (The other cases can be easily checked).

From relation (9) we obtain that

$$
\begin{aligned}
{\left[x^{n} y^{r} t^{\gamma}\right] F_{r v}=} & \left(a_{n, r, \gamma+1}+a_{n-1, r, \gamma}-a_{n-1, r, \gamma+1}\right) k^{n-2 r} \\
= & \frac{1}{r}\left[\left(\begin{array}{c}
n-r+1 \\
r+1
\end{array}\right)\left(\begin{array}{c}
r \\
\gamma+1
\end{array}\right)\left(\begin{array}{c}
n-r \\
\gamma
\end{array}\right)+\left(\begin{array}{c}
n-r \\
r+1
\end{array}\right)\left(\begin{array}{c}
r \\
\gamma
\end{array}\right)\left(\begin{array}{c}
n-r-1 \\
\gamma-1
\end{array}\right)-\right. \\
& \left.\left(\begin{array}{c}
n-r \\
r+1
\end{array}\right)\left(\begin{array}{c}
r \\
\gamma+1
\end{array}\right)\left(\begin{array}{c}
n-r-1 \\
\gamma
\end{array}\right)\right] k^{n-2 r}
\end{aligned}
$$

giving, after some simple manipulations, that

$$
\left[x^{n} y^{r} t^{\gamma}\right] F_{r v}=\frac{1}{n-r}\left(\begin{array}{c}
n-r \\
r
\end{array}\right)\left(\begin{array}{l}
r \\
\gamma
\end{array}\right)\left(\begin{array}{c}
n-r \\
\gamma+1
\end{array}\right) k^{n-2 r}
$$

We now come to the parameters $d r$ and $p$. We first need the following result, the proof of which is straightforward and it is omitted.

Lemma 3.5 If $q_{1}, q_{2}$ are two parameters of $\mathcal{M}$ with

$$
q_{1}(u)+q_{2}(u)=r(u), \quad \text { for each } u \in \mathcal{M}
$$

then $F_{q_{2}}(x, y, t)=F_{q_{1}}\left(x, y t, t^{-1}\right)$. Hence, $\left[x^{n} y^{r} t^{\gamma}\right] F_{q_{2}}=\left[x^{n} y^{r} t^{r-\gamma}\right] F_{q_{1}}$.

Clearly, each of the pairs $\{l p, d r\}$ and $\{l d r, p\}$ satisfies relation (10), so that from Propositions 3.1, 3.3 and 3.4 we obtain the following result.

Proposition 3.6 The number of all $u \in \mathcal{M}_{n, r}$ with $\gamma$ double rises and the number of all $u \in \mathcal{M}_{n, r}$ with $\gamma$ peaks are equal respectively to

$$
\left[x^{n} y^{r} t^{\gamma}\right] F_{d r}=\frac{1}{r}\left(\begin{array}{c}
n-r+1 \\
n-2 r
\end{array}\right)\left(\begin{array}{l}
r \\
\gamma
\end{array}\right)\left(\begin{array}{c}
n-r \\
r-\gamma-1
\end{array}\right) k^{n-2 r}
$$

where $0 \leq \gamma \leq r-1$ and

$$
\left[x^{n} y^{r} t^{\gamma}\right] F_{p}=\frac{1}{n-r}\left(\begin{array}{c}
n-r \\
r
\end{array}\right)\left(\begin{array}{c}
r \\
\gamma
\end{array}\right)\left(\begin{array}{c}
n-r \\
r-\gamma+1
\end{array}\right) k^{n-2 r}
$$

where $0 \leq \gamma \leq r$.

Remark Notice that if we apply the above formulae for $n=2 r$ we obtain that the number of all Dyck paths with semilength $r$ and $\gamma$ double rises (resp. peaks) is equal to the Narayana number $\frac{1}{r}\left(\begin{array}{c}r \\ \gamma\end{array}\right)\left(\begin{array}{c}r \\ \gamma+1\end{array}\right)$ (resp. $\left.\frac{1}{r}\left(\begin{array}{c}r \\ \gamma-1\end{array}\right)\left(\begin{array}{c}r \\ \gamma\end{array}\right)\right)$ (see, for example, [5]). 


\section{Low peaks and low valleys}

A peak (resp. valley) at height 1 (resp. 0) is called low peak (resp. low valley). We denote by $\check{p}$ (resp. $\check{v}$ ) the parameter of $\mathcal{M}$ determined by the number of low peaks (resp. low valleys). Low left and low right peaks and valleys, as well as the parameters induced by them are defined similarly. Since each of the pairs $\{\check{l p}, \check{r p}\}$ and $\{\check{l v}, \check{r v}\}$ consists of equidistributed parameters, it is sufficient to consider $\check{q}$ when $q \in\{l p, p, v, r v\}$.

For every such $q$ we consider the set $N_{\check{q}}$ of all $\check{q}$-free $k$-colored Motzkin paths i.e.,

$$
\mathcal{N}_{\check{q}}=\{u \in \mathcal{M}: \check{q}(u)=0\}
$$

and its generating function $G_{\breve{q}}(x, y)$ according to length and number of rises i.e.,

$$
G_{\breve{q}}(x, y)=\sum_{u \in \mathcal{N}_{\breve{q}}} x^{l(u)} y^{r(u)} .
$$

In the sequel we find for each $\check{q}$ the formula of $G_{\check{q}}$, which is used to obtain the generating function $F_{\check{q}}$.

We start with the parameter $\check{l p}$.

If $u \in \mathcal{N}_{\check{l p}}$ then $u=\epsilon$, or $u=\beta_{\nu} z$, or $u=a w \bar{a} z$ where $w \in \mathcal{A}, z \in \mathcal{N}_{\check{l p}}$ and $\nu \in[k]$.

It follows that

$$
G_{\check{l p}}(x, y)=1+k x G_{\check{l p}}(x, y)+x^{2} y A(x, y) G_{\check{l p}}(x, y)
$$

and since $A(x, y)=x^{2} y F^{2}(x, y)$, we finally obtain that

$$
G_{\check{l p}}(x, y)=\frac{1}{1-k x-x^{4} y^{2} F^{2}(x, y)}
$$

For the next result, we use the double sequence $b_{n, m}$ of ballot numbers ([16], p.130), defined by $b_{0,0}=1$ and $b_{n, m}=\left(\begin{array}{c}n+m \\ m\end{array}\right)-\left(\begin{array}{c}n+m \\ m-1\end{array}\right)=\frac{n+1-m}{n+1}\left(\begin{array}{c}n+m \\ m\end{array}\right)$ and the following variation of the Vandermonde convolution formula ([10], (3.2))

$$
\sum_{\nu=0}^{n}\left(\begin{array}{c}
\alpha+\nu \\
\nu
\end{array}\right)\left(\begin{array}{c}
\beta+n-\nu \\
n-\nu
\end{array}\right)=\left(\begin{array}{c}
\alpha+\beta+n+1 \\
n
\end{array}\right)
$$

Lemma 4.1 For every $n, r, s, \rho \in \mathbb{N}$ we have,

$$
\left[x^{n} y^{r}\right] G_{l p}^{s+1} F^{\rho}=k^{n-2 r} \sum_{\nu=0}^{\left[\frac{r}{2}\right]}\left(\begin{array}{c}
s+\nu \\
\nu
\end{array}\right)\left(\begin{array}{c}
n+\rho+s-\nu \\
n-2 r
\end{array}\right) b_{r+\rho-1, r-2 \nu} .
$$

Proof: For $\rho \neq 0$, using relation (11), as well as (2) and (12), we have that

$$
G_{\check{l p}}^{s+1}(x, y) F^{\rho}(x, y)=\sum_{m=0}^{\infty}(-1)^{m}\left(\begin{array}{c}
-(s+1) \\
m
\end{array}\right)\left(k+x^{3} y^{2} F^{2}(x, y)\right)^{m} x^{m} F^{\rho}(x, y)
$$




$$
\begin{aligned}
= & \sum_{m=0}^{\infty} \sum_{\nu=0}^{m}\left(\begin{array}{c}
s+m \\
m
\end{array}\right)\left(\begin{array}{c}
m \\
\nu
\end{array}\right) k^{m-\nu} x^{3 \nu+m} y^{2 \nu} F^{2 \nu+\rho}(x, y) \\
= & \sum_{m=0}^{\infty} \sum_{\nu=0}^{m} \sum_{\sigma=0}^{\infty} \sum_{\lambda=0}^{\left[\frac{\sigma}{2}\right]}\left(\begin{array}{c}
s+\nu \\
\nu
\end{array}\right)\left(\begin{array}{c}
m+s \\
\nu+s
\end{array}\right) \frac{2 \nu+\rho}{\sigma+2 \nu+\rho} . \\
& \cdot\left(\begin{array}{c}
\sigma+2 \nu+\rho \\
2 \nu+\rho+\lambda, \lambda, \sigma-2 \lambda
\end{array}\right) k^{m-\nu+\sigma-2 \lambda} x^{3 \nu+m+\sigma} y^{2 \nu+\lambda} \\
= & \sum_{n=0}^{\infty} \sum_{r=0}^{\left[\frac{n}{2}\right]} \sum_{\nu=0}^{\left[\frac{r}{2}\right]} \sum_{i=0}^{n-2 r}\left(\begin{array}{c}
s+\nu \\
\nu
\end{array}\right)\left(\begin{array}{c}
n-2 r+\nu+s-i \\
\nu+s
\end{array}\right) . \\
& \cdot \frac{2 \nu+\rho}{i-2 \nu+2 r+\rho}\left(\begin{array}{c}
i-2 \nu+2 r+\rho \\
r+\rho, r-2 \nu, i
\end{array}\right) x^{n} y^{r} k^{n-2 r} .
\end{aligned}
$$

It follows that

$$
\begin{aligned}
{\left[x^{n} y^{r}\right] G^{s+1} F^{\rho}=} & \sum_{\nu=0}^{\left[\frac{r}{2}\right]}\left(\begin{array}{c}
s+\nu \\
\nu
\end{array}\right) \frac{2 \nu+\rho}{2 r-2 \nu+\rho}\left(\begin{array}{c}
2 r-2 \nu+\rho \\
r+\rho
\end{array}\right) . \\
& \cdot \sum_{i=0}^{n-2 r}\left(\begin{array}{c}
n-2 r+\nu+s-i \\
n-2 r-i
\end{array}\right)\left(\begin{array}{c}
2 r-2 \nu-1+\rho+i \\
i
\end{array}\right) k^{n-2 r} \\
= & \sum_{\nu=0}^{\left[\frac{r}{2}\right]}\left(\begin{array}{c}
s+\nu \\
\nu
\end{array}\right)\left(\begin{array}{c}
n+s+\rho-\nu \\
n-2 r
\end{array}\right) b_{r+\rho-1, r-2 \nu} k^{n-2 r} .
\end{aligned}
$$

The proof for the case $\rho=0$ is similar and it is omitted.

Remark Notice that for $s=\rho=0$ and $n=2 r$ we obtain that the number of all $u \in \mathcal{D}_{r}$ with no low peaks is equal to the Fine number $(\mathrm{A} 000957) f_{r}=\sum_{\nu=0}^{\left[\frac{r}{2}\right]} b_{r-1, r-2 \nu}=$ $\sum_{\nu=0}^{\left[\frac{r}{2}\right]} \frac{\nu}{r-\nu}\left(\begin{array}{c}2 r-2 \nu \\ r\end{array}\right)([7],(\mathrm{C} .6))$.

We now proceed to determine $F_{\check{l p}}$. It is clear that every $u \in \mathcal{M}$ can be written uniquely in one of the forms $u=w$, or $u=w a \bar{a} z$, or $u=w a \beta_{\nu} \tau \bar{a} z$ where $w \in \mathcal{N}_{\check{l p}}, z, \tau \in \mathcal{M}$ and $\nu \in[k]$. Clearly, since in the second and third case $\check{l p}(u)=\check{l p}(z)+1$, we obtain that

$$
F_{\check{l p}}(x, y, t)=G_{\check{l p}}(x, y)+x^{2} y t G_{\check{l p}}(x, y) F_{\check{l p}}(x, y, t)+k x^{3} y t G_{\check{l p}}(x, y) F(x, y) F_{\check{l p}}(x, y, t)
$$

and hence

$$
F_{\check{l p}}(x, y, t)=\frac{G_{\check{l p}}(x, y)}{1-x^{2} y t(1+k x F(x, y)) G_{\check{l p}}(x, y)}
$$

We have the following result. 
Proposition 4.2 The number of all $u \in \mathcal{M}_{n, r}$ with $\gamma$ low left peaks is given by the formula

$$
\left[x^{n} y^{r} t^{\gamma}\right] F_{\check{l p}}=k^{n-2 r} \sum_{\rho=0}^{n-2 r} \sum_{\nu=0}^{\left[\frac{r-\gamma}{2}\right]}\left(\begin{array}{c}
\gamma \\
\rho
\end{array}\right)\left(\begin{array}{c}
\gamma+\nu \\
\nu
\end{array}\right)\left(\begin{array}{c}
n-\gamma-\nu \\
n-2 r-\rho
\end{array}\right) b_{r-\gamma+\rho-1, r-\gamma-2 \nu},
$$

where $0 \leq \gamma \leq r$.

Proof: Using lemma 4.1 and relation (13) we obtain that

$$
\begin{aligned}
& F_{\check{l p}}(x, y, t)=\sum_{\gamma=0}^{\infty}(1+k x F(x, y))^{\gamma} G^{\gamma+1}(x, y) x^{2 \gamma} y^{\gamma} t^{\gamma} \\
&=\sum_{\gamma=0}^{\infty} \sum_{\rho=0}^{\gamma}\left(\begin{array}{c}
\gamma \\
\rho
\end{array}\right) G_{\check{l p}}^{\gamma+1}(x, y) F^{\rho}(x, y) k^{\rho} x^{2 \gamma+\rho} y^{\gamma} t^{\gamma} \\
&=\sum_{\gamma=0}^{\infty} \sum_{\rho=0}^{\gamma} \sum_{\sigma=0}^{\infty} \sum_{\lambda=0}^{\left[\frac{\sigma}{2}\right]} \sum_{\nu=0}^{\left[\frac{\lambda}{2}\right]}\left(\begin{array}{c}
\gamma \\
\rho
\end{array}\right)\left(\begin{array}{c}
\gamma+\nu \\
\nu
\end{array}\right)\left(\begin{array}{c}
\sigma+\rho+\gamma-\nu \\
\sigma-2 \lambda
\end{array}\right) . \\
&=\sum_{n=0}^{\infty} \sum_{r=0}^{\left[\frac{n}{2}\right]} \sum_{\gamma=0}^{r} \sum_{\rho=0}^{n-2 r} \sum_{\nu=0}^{\left.\frac{r-\gamma}{2}\right]}\left(\begin{array}{c}
\gamma \\
\rho
\end{array}\right)\left(\begin{array}{c}
\gamma+\nu \\
\nu
\end{array}\right)\left(\begin{array}{c}
n-\gamma-\nu \\
n-2 r-\rho
\end{array}\right) . \\
& b_{r-\gamma+\rho-1, r-\gamma-2 \nu} k^{n-2 r} x^{n} y^{r} t^{\gamma},
\end{aligned}
$$

giving the required result.

Remark Notice that for $n=2 r$ we obtain formula (6.16) of [7] on Dyck paths with $\gamma$ low peaks.

We now come to the parameter $\check{p}$.

Clearly if $u \in \mathcal{N}_{\check{p}}$ then $u=\epsilon$, or $u=\beta_{\nu} z$, or $u=a w \bar{a} z$ where $w \in \mathcal{M} \backslash\{\epsilon\}, z \in \mathcal{N}_{\check{p}}$ and $\nu \in[k]$.

It follows that

$$
G_{\check{p}}(x, y)=1+k x G_{\check{p}}(x, y)+x^{2} y(F(x, y)-1) G_{\check{p}}(x, y)
$$

and using equation (1) we obtain that

$$
G_{\check{p}}(x, y)=\frac{F(x, y)}{1+x^{2} y F(x, y)}
$$

We now have the following result.

Proposition 4.3 The number of all $u \in \mathcal{M}_{n, r}$ with $\gamma$ low peaks is given by the formula

$$
\left[x^{n} y^{r} t^{\gamma}\right] F_{\breve{p}}=\frac{\gamma+1}{r+1} k^{n-2 r} \sum_{\lambda=0}^{r-\gamma}(-1)^{r-\gamma-\lambda}\left(\begin{array}{c}
r-\lambda+1 \\
\gamma+1
\end{array}\right)\left(\begin{array}{c}
n-r+\lambda \\
r, \lambda, n-2 r
\end{array}\right),
$$

where $0 \leq \gamma \leq r$ 
Proof: Every $u \in \mathcal{M}$ can be uniquely written in either of the forms $u=w$, or $u=w a \bar{a} z$ where $w \in \mathcal{N}_{\check{p}}$ and $z \in \mathcal{M}$, with $\check{p}(u)=\check{p}(z)+1$. It follows that

$$
F_{\check{p}}(x, y, t)=G_{\check{p}}(x, y)+x^{2} y t G_{\check{p}}(x, y) F_{\check{p}}(x, y, t) .
$$

Thus, from relation (14) follows that

$$
F_{\check{p}}(x, y, t)=\frac{F(x, y)}{1-x^{2} y(t-1) F(x, y)} .
$$

Furthermore, using relation (2) we obtain that

$$
\begin{aligned}
F_{\breve{p}}(x, y, t) & =\sum_{m=0}^{\infty} F^{m+1}(x, y)(t-1)^{m} x^{2 m} y^{m} \\
= & \sum_{m=0}^{\infty} \sum_{\sigma=0}^{\infty} \sum_{\lambda=0}^{\left[\frac{\sigma}{2}\right]} \sum_{\gamma=0}^{m}(-1)^{m-\gamma} \frac{m+1}{\sigma+m+1}\left(\begin{array}{c}
m \\
\gamma
\end{array}\right)\left(\begin{array}{c}
\sigma+m+1 \\
\lambda+m+1, \lambda, \sigma-2 \lambda
\end{array}\right) k^{\sigma-2 \lambda} x^{2 m+\sigma} y^{m+\lambda} t^{\gamma} \\
= & \sum_{n=0}^{\infty} \sum_{r=0}^{\left[\frac{n}{2}\right]} \sum_{\gamma=0}^{r} \sum_{\lambda=0}^{r-\gamma}(-1)^{r-\gamma-\lambda} \frac{r-\lambda+1}{n-r+\lambda+1}\left(\begin{array}{c}
r-\lambda \\
\gamma
\end{array}\right)\left(\begin{array}{c}
n-r+\lambda+1 \\
r+1, \lambda, n-2 r
\end{array}\right) k^{n-2 r} x^{n} y^{r} t^{\gamma} .
\end{aligned}
$$

giving the required result.

Remark Notice that for $n=2 r$ we deduce that the number of all $u \in \mathcal{D}_{r}$ with $\gamma$ low peaks is equal to $\frac{\gamma+1}{r+1} \sum_{\lambda=0}^{r-\gamma}(-1)^{r-\gamma-\lambda}\left(\begin{array}{c}r-\lambda+1 \\ \gamma+1\end{array}\right)\left(\begin{array}{c}r+\lambda \\ r\end{array}\right)$, thus obtaining a formula equivalent to (6.16) of [7].

Also notice that if, in addition, $\gamma=0$, then we obtain the Fine numbers, as in relation (C.5) of [7].

Next we deal with the parameter $\check{v}$.

It is clear that every $u \in \mathcal{N}_{\check{u}}$ can be uniquely written in the form $u=u^{\prime} u^{\prime \prime}$, where $u^{\prime} \in\{\epsilon\} \cup\{a w \bar{a}: w \in \mathcal{M}\}$ and $u^{\prime \prime} \in\{\epsilon\} \cup\left\{\beta_{\nu} z: z \in \mathcal{N}_{\check{v}}\right.$ and $\left.\nu \in[k]\right\}$.

It follows that

$$
G_{\check{v}}(x, y)=\left(1+x^{2} y F(x, y)\right)\left(1+k x G_{\check{v}}(x, y)\right)
$$

and hence

$$
G_{\check{v}}(x, y)=\frac{1+x^{2} y F(x, y)}{1-k x-k x^{3} y F(x, y)}
$$

Now, since each $u \in \mathcal{M}$ can be written uniquely in one of the forms $u=\tau$, or $u=a w \bar{a} z$, or $u=\tau \beta_{\nu} a w \bar{a} z$ where $\tau \in \mathcal{N}_{\check{u}}, w \in \mathcal{M}, z \in \mathcal{A}$ and $\nu \in[k]$, we can easily deduce from relation (15) that

$$
\begin{aligned}
F_{\breve{v}}(x, y, t) & =G_{\check{v}}(x, y)+x^{2} y t F(x, y) A_{\breve{v}}(x, y, t)+k x^{3} y t G_{\check{v}}(x, y) F(x, y) A_{\breve{v}}(x, y, t) \\
& =G_{\breve{v}}(x, y)+x^{2} y t F(x, y)\left(1+k x G_{\check{v}}(x, y)\right)\left(F_{\check{v}}(x, y, t)-1-k x F_{\check{v}}(x, y, t)\right)
\end{aligned}
$$


which gives

$$
\begin{aligned}
F_{\breve{v}}(x, y, t) & =\frac{G_{\breve{v}}(x, y)-x^{2} y t F(x, y)\left(1+k x G_{\check{v}}(x, y)\right)}{1-x^{2} y t F(x, y)\left(1+k x G_{\check{v}}(x, y)\right)(1-k x)} \\
& =\frac{1+x^{2} y F(x, y)-x^{2} y t F(x, y)}{1-k x-x^{2} y t F(x, y)+k x^{3} y t F(x, y)-k x^{3} y F(x, y)} .
\end{aligned}
$$

Thus,

$$
F_{\check{v}}(x, y, t)=\frac{g(x, y, t)}{1-\left(k x g(x, y, t)+x^{2} y t F(x, y)\right)}
$$

where $g(x, y, t)=1+x^{2} y(1-t) F(x, y)$.

Using relation (16), as well as (2), (4), a version of (12) ([10], (3.3)) and combinatorial calculus similar to that of the proofs of propositions 4.2 and 4.3 we obtain the following result.

Proposition 4.4 The number of all $u \in \mathcal{M}_{n, r}$ with $\gamma$ low valleys is given by the formula

$$
\left[x^{n} y^{r} t^{\gamma}\right] F_{\check{v}}=\frac{\gamma+1}{r} k^{n-2 r} \sum_{\rho=0}^{\min \{n-2 r, r-\gamma-1\}}\left(\begin{array}{c}
\gamma+1+\rho \\
\rho
\end{array}\right)\left(\begin{array}{c}
2 r-\gamma-2-\rho \\
r-1
\end{array}\right)\left(\begin{array}{c}
n-\gamma-\rho \\
2 r-\gamma
\end{array}\right)
$$

where $0 \leq \gamma \leq r-1$

Remark Notice that for $n=2 r$ we deduce that the number of all $u \in \mathcal{D}_{r}$ with $\gamma$ low valleys is equal to $\frac{\gamma+1}{2 r-\gamma-1}\left(\begin{array}{c}2 r-\gamma-1 \\ r\end{array}\right),([7],(6.34))$.

Finally, for the parameter $\check{r v}$, it is clear that every $u \in \mathcal{N}_{\check{r v}}$ can be uniquely written in the form $u=u^{\prime} u^{\prime \prime}$, where $u^{\prime} \in\{\epsilon\} \cup\{a w \bar{a}: w \in \mathcal{M}\}$ and $u^{\prime \prime} \in\left\{\beta_{1}, \beta_{2}, \ldots, \beta_{k}\right\}^{*}$.

It follows that

$$
G_{\check{r v}}(x, y)=\frac{1+x^{2} y F(x, y)}{1-k x}
$$

Since each $u \in \mathcal{M}$ is written uniquely in either of the forms $u=\tau$ or $u=w z$, where $\tau \in \mathcal{N}_{\text {řv }}, w \in \mathcal{N}_{\text {řv }} \backslash\{\epsilon\}$ and $z \in \mathcal{A}$ it follows that

$$
F_{\check{r v}}(x, y, t)=G_{r \check{v v}}(x, y)+t\left(G_{\check{r v}}(x, y)-1\right) A_{\check{r v}}(x, y, t)
$$

Furthermore, we can easily check that

$$
B_{r \check{v}}(x, y, t)=1+k x B_{\check{r v}}(x, y, t)+k x t A_{\check{r v}}(x, y, t)
$$

so that

$$
(1-k x)\left(F_{r \check{v}}(x, y, t)-A_{r \check{r v}}(x, y, t)\right)=1+k x t A_{r \check{r v}}(x, y, t)
$$

and

$$
A_{\check{r v}}(x, y, t)=\frac{(1-k x) F_{r \check{v}}(x, y, t)-1}{1-k x+k x t}
$$


By relations (17), (18) and (19), it follows easily that

$$
F_{r \check{v}}(x, y, t)=\frac{1+x^{2} y(1-t) F(x, y)}{1-\left(k x+x^{2} y t F(x, y)\right)}
$$

Furthermore, using relations (20) and (12) and proceeding in a way similar to that of the proofs of Propositions 4.2 and 4.3 we obtain the following result.

Proposition 4.5 The number of all $u \in \mathcal{M}_{n, r}$ with $\gamma$ low right valleys is equal to

$$
\left[x^{n} y^{r} t^{\gamma}\right] F_{\check{r v}}=\left[\frac{\gamma}{2 r-\gamma}\left(\begin{array}{c}
2 r-\gamma \\
r
\end{array}\right)\left(\begin{array}{c}
n-1 \\
2 r
\end{array}\right)+\frac{\gamma+1}{2 r-\gamma-1}\left(\begin{array}{c}
2 r-\gamma-1 \\
r
\end{array}\right)\left(\begin{array}{c}
n-1 \\
2 r-1
\end{array}\right)\right] k^{n-2 r}
$$

where $0 \leq \gamma \leq r-1$.

Remark We note that for $n=2 r$ we deduce that the number of all $u \in \mathcal{D}_{r}$ with $\gamma$ low (right) valleys is equal to $\frac{\gamma+1}{2 r-\gamma-1}\left(\begin{array}{c}2 r-\gamma-1 \\ r\end{array}\right)$ [7].

\section{$5 \quad$ High peaks and high valleys}

A peak (resp. valley) that is not low is called high peak (resp. high valley). The parameters $\hat{p}=p-\check{p}$ and $\hat{v}=v-\check{v}$ determine the number of high peaks and high valleys, respectively. High left and high right peaks and valleys, as well as the corresponding parameters are defined similarly. As in the case of low peaks and low valleys, it is sufficient to restrict ourselves to the parameters $\{\hat{l p}, \hat{p}, \hat{v}, \hat{r} v\}$. For any such parameter $q$ we have:

$$
B_{\hat{q}}(x, y, t)=1+k x F_{\hat{q}}(x, y, t)
$$

and

$$
A_{\hat{q}}(x, y, t)=x^{2} y F_{q}(x, y, t) F_{\hat{q}}(x, y, t)
$$

It follows that

$$
F_{\hat{q}}(x, y, t)=1+k x F_{\hat{q}}(x, y, t)+x^{2} y F_{q}(x, y, t) F_{\hat{q}}(x, y, t)
$$

and hence

$$
F_{\hat{q}}(x, y, t)=\frac{1}{1-\left(k x+x^{2} y F_{q}(x, y, t)\right)}
$$

Using a similar technique to that of the proof of relation (6), we can find formulae for the powers $F_{q}^{s}, s \in \mathbb{N}^{*}$, where $q \in\{r v, v, p\}$ :

$$
\left[x^{n} y^{r} t^{\gamma}\right] F_{r v}^{s}=\frac{s}{n-r}\left(\begin{array}{c}
n-r \\
r
\end{array}\right)\left(\begin{array}{l}
r \\
\gamma
\end{array}\right)\left(\begin{array}{c}
n-r+s-1 \\
\gamma+s
\end{array}\right) k^{n-2 r}
$$

where $0 \leq \gamma \leq r$ 


$$
\left[x^{n} y^{r} t^{\gamma}\right] F_{v}^{s}=\frac{s}{r}\left(\begin{array}{c}
n-r+s \\
n-2 r
\end{array}\right)\left(\begin{array}{l}
r \\
\gamma
\end{array}\right)\left(\begin{array}{c}
n-r+s-1 \\
r-\gamma-1
\end{array}\right) k^{n-2 r}
$$

where $0 \leq \gamma \leq r-1$.

$$
\left[x^{n} y^{r} t^{\gamma}\right] F_{p}^{s}=\frac{s}{n-r}\left(\begin{array}{c}
n-r \\
r
\end{array}\right)\left(\begin{array}{l}
r \\
\gamma
\end{array}\right)\left(\begin{array}{c}
n-r+s-1 \\
r-\gamma+s
\end{array}\right) k^{n-2 r}
$$

where $0 \leq \gamma \leq r$

After expanding $F_{\hat{q}}(x, y, t)$ into a geometric series and using the previous relations we will obtain the corresponding enumeration results for $\hat{p}, \hat{l p}, \hat{v}$ and $\hat{r v}$.

More precisely, for the parameter $\hat{p}$ we have the following result.

Proposition 5.1 The number of all $u \in \mathcal{M}_{n, r}$ with $\gamma$ high peaks is equal to

$$
\left[x^{n} y^{r} t^{\gamma}\right] F_{\hat{p}}=\frac{1}{r}\left(\begin{array}{c}
n-r+1 \\
n-2 r
\end{array}\right)\left(\begin{array}{l}
r \\
\gamma
\end{array}\right)\left(\begin{array}{c}
n-r \\
r-\gamma-1
\end{array}\right) k^{n-2 r}
$$

where $0 \leq \gamma \leq r-1$.

Proof: Using relations (21) and (24) we obtain that

$$
\begin{aligned}
F_{\hat{p}}(x, y, t)= & \sum_{m=0}^{\infty}\left(k+x y F_{p}(x, y, t)\right)^{m} x^{m} \\
= & \sum_{m=0}^{\infty} \sum_{\nu=0}^{\infty}\left(\begin{array}{c}
m \\
\nu
\end{array}\right) F_{p}^{\nu}(x, y, t) k^{m-\nu} x^{m+\nu} y^{\nu} \\
= & \sum_{m=0}^{\infty} k^{m} x^{m}+\sum_{m=1}^{\infty} \sum_{\nu=1}^{\infty} \sum_{\sigma=0}^{\infty} \sum_{\lambda=0}^{\left[\frac{\sigma}{2}\right]} \sum_{\gamma=0}^{\lambda}\left(\begin{array}{c}
m \\
\nu
\end{array}\right) \frac{\nu}{\sigma-\lambda}\left(\begin{array}{c}
\sigma-\lambda \\
\lambda
\end{array}\right)\left(\begin{array}{c}
\lambda \\
\gamma
\end{array}\right) . \\
& \cdot\left(\begin{array}{c}
\sigma-\lambda+\nu-1 \\
\lambda-\gamma+\nu
\end{array}\right) k^{m-\nu+\sigma-2 \lambda} x^{m+\nu+\sigma} y^{\nu+\lambda} t^{\gamma} \\
& \sum_{n=0}^{\infty} k^{n} x^{n}+\sum_{n=0}^{\infty} \sum_{r=0}^{\left[\frac{n}{2}\right]} \sum_{\gamma=0}^{r-1} \sum_{\rho=0}^{n-2 r} \sum_{\nu=1}^{r-\gamma} \frac{\nu+r-\nu}{\rho+}\left(\begin{array}{c}
n-2 r+\nu-\rho \\
\nu
\end{array}\right) . \\
& \quad\left(\begin{array}{c}
\rho+r-\nu \\
\rho
\end{array}\right)\left(\begin{array}{c}
r+r-1 \\
\gamma-\gamma
\end{array}\right) k^{n-2 r} x^{n} y^{r} t^{\gamma}
\end{aligned}
$$

Thus, for $0 \leq \gamma \leq r-1$ and using relation (12) we have that

$$
\begin{aligned}
& {\left[x^{n} y^{r} t^{\gamma}\right] F_{\hat{p}}=\sum_{\rho=0}^{n-2 r} \frac{(n-2 r-\rho+1)\left(\begin{array}{c}
\rho+r-1 \\
r-\gamma
\end{array}\right)(\rho+\gamma-1) !}{\rho ! \gamma !} } \\
& \cdot \sum_{\nu=1}^{r-\gamma}\left(\begin{array}{c}
n-2 r+\nu-\rho \\
\nu-1
\end{array}\right)\left(\begin{array}{c}
\rho+r-\nu-1 \\
r-\nu-\gamma
\end{array}\right) k^{n-2 r}
\end{aligned}
$$

THE ELECTRONic Journal of COMBinatorics 12 (2005), \#R16 


$$
\begin{aligned}
& =\frac{1}{r}\left(\begin{array}{l}
r \\
\gamma
\end{array}\right) \sum_{\rho=0}^{n-2 r}(n-2 r-\rho+1)\left(\begin{array}{c}
\rho+r-1 \\
\rho
\end{array}\right) \cdot \\
& =\frac{1}{r}\left(\begin{array}{l}
r \\
\gamma
\end{array}\right) \sum_{\rho=0}^{r-\gamma-1}\left(\begin{array}{c}
n-2 r-\rho+1+\nu \\
\nu
\end{array}\right)\left(\begin{array}{c}
\rho+r-2-\nu \\
r-\gamma-1-\nu
\end{array}\right) k^{n-2 r} \\
& =\frac{1}{r}\left(\begin{array}{l}
r \\
\gamma
\end{array}\right)\left(\begin{array}{c}
n-2 r-\rho \\
n-r+1 \\
n-2 r
\end{array}\right)\left(\begin{array}{c}
n-r \\
r-\gamma-1
\end{array}\right) k^{n-2 r} .
\end{aligned}
$$

In the following result we consider the remaining three cases. Though the produced formulae are more complicated, their proofs are similar to the proof of relation (25) and are omitted.

Proposition 5.2 The number of all $u \in \mathcal{M}_{n, r}$ with $\gamma$ high left peaks, high valleys and high right valleys is given respectively by the formulae

$$
\left[x^{n} y^{r} t^{\gamma}\right] F_{l p}=\frac{1}{r} k^{n-2 r} \sum_{\rho=0}^{n-2 r}(n-2 r-\rho+1)\left(\begin{array}{c}
n-r-\rho \\
r-\gamma-1
\end{array}\right)\left(\begin{array}{c}
\rho+r \\
\gamma
\end{array}\right)\left(\begin{array}{c}
\rho+r-1 \\
\rho
\end{array}\right)
$$

where $0 \leq \gamma \leq r-1$,

$$
\begin{array}{r}
{\left[x^{n} y^{r} t^{\gamma}\right] F_{\hat{v}}=\left[\delta_{\gamma 0}\left(\begin{array}{c}
n \\
2 r
\end{array}\right)+\sum_{\rho=0}^{n-2 r} \sum_{\nu=1}^{r-\gamma-1} \frac{\nu}{r-\nu}\left(\begin{array}{c}
n-2 r-\rho+\nu \\
\nu
\end{array}\right)\left(\begin{array}{c}
r-\nu \\
\gamma
\end{array}\right) \cdot\right.} \\
\left.\cdot\left(\begin{array}{c}
\rho+r \\
\rho
\end{array}\right)\left(\begin{array}{c}
\rho+r-1 \\
\rho+\gamma+\nu
\end{array}\right)\right] k^{n-2 r}
\end{array}
$$

where $0 \leq \gamma \leq r-2$,

$$
\begin{array}{r}
{\left[x^{n} y^{r} t^{\gamma}\right] F_{r \hat{v}}=\left[\delta_{\gamma 0}\left(\begin{array}{c}
n \\
2 r
\end{array}\right)+\sum_{\rho=0}^{n-2 r} \sum_{\nu=1}^{\min \{r-\gamma, r-1\}} \frac{\nu}{r-\nu}\left(\begin{array}{c}
n-2 r-\rho+\nu \\
\nu
\end{array}\right)\left(\begin{array}{c}
r-\nu \\
\gamma
\end{array}\right) .\right.} \\
\left.\cdot\left(\begin{array}{c}
\rho+r-\nu-1 \\
\rho
\end{array}\right)\left(\begin{array}{c}
\rho+r-1 \\
\gamma+\nu
\end{array}\right)\right] k^{n-2 r}
\end{array}
$$

where $0 \leq \gamma \leq r-1$. (Here $\delta$ is the Kronecker symbol).

Notice that for $n=2 r$, we obtain from (26), or (27) (resp. (28), or (29)) the corresponding result for high peaks (resp. high valleys) in Dyck paths [7].

We note that from Propositions 3.1 (ii), 3.6 and 5.1 we obtain the following result, which is well-known in the case of Dyck paths [6]. 
Proposition 5.3 The parameters $\hat{p}$ and $v$ of $\mathcal{M}$ are equidistributed.

We close by presenting a combinatorial proof of the above result. For this we need the following lemma which extends a result for Dyck paths [12] to $k$-colored Motzkin paths.

Lemma 5.4 For each $i \in \mathbb{N}$ there exists an involution $\psi_{i}$ of $\mathcal{M}$ such that

$$
p_{i+2}(u)=v_{i}\left(\psi_{i}(u)\right)
$$

where $p_{i}(u), v_{i}(u)$ denote respectively the number of peaks and valleys of $u$ at height $i$.

Proof: For each path $u \in \mathcal{M}$ we define the path $\psi_{i}(u)$, by turning each peak $(j, i+2)$ of $u$ into a valley $(j, i)$ and each valley $(j, i)$ of $u$ into a peak $(j, i+2)$. The remaining points of $u$ are fixed; see Fig. 3 .
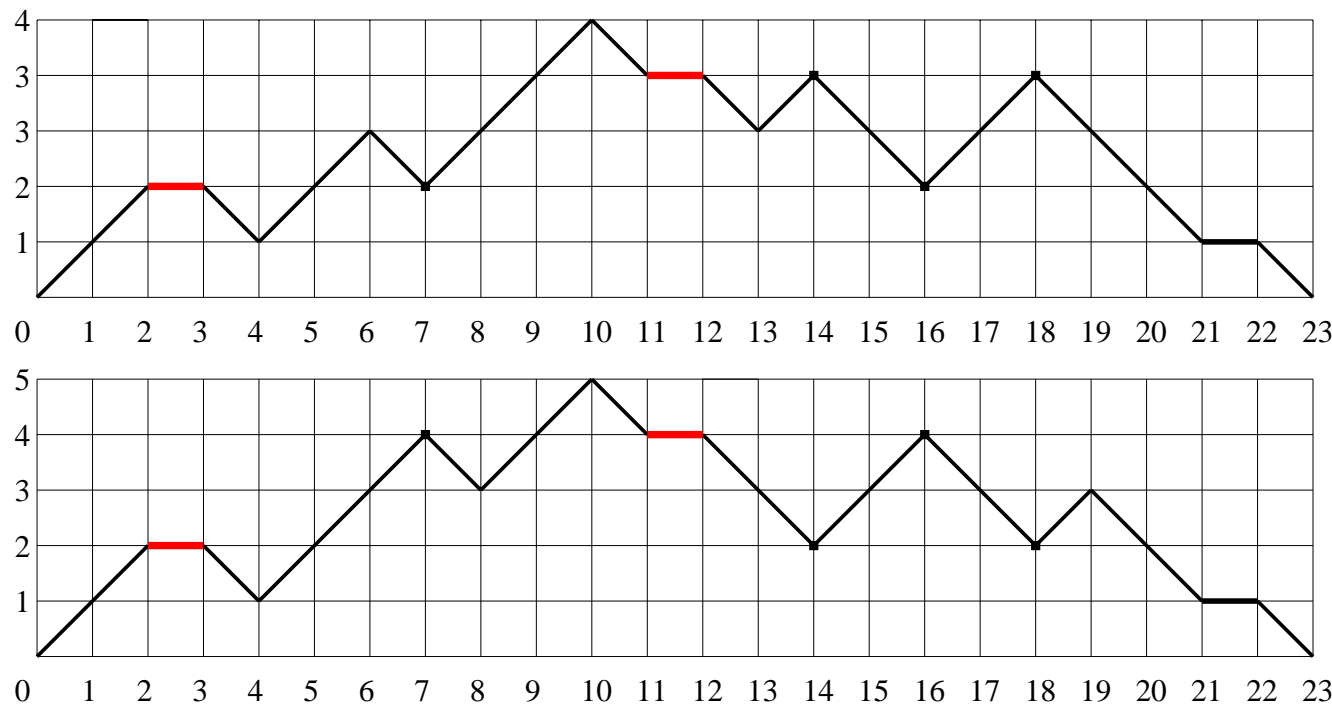

Figure 3: The paths $u$ and $\psi_{2}(u)$

Proof of Proposition 5.3: It is sufficient to find a bijection

such that

$$
\psi: \mathcal{M}_{n, r} \rightarrow \mathcal{M}_{n, r}
$$

$$
\hat{p}(u)=v(\psi(u)), \text { for each } u \in \mathcal{M}_{n, r} .
$$

We assume that $r \geq 1$; (in the trivial case $r=0, \psi$ is the identity mapping).

We define

$$
\psi(u)=\left(\psi_{l} \circ \psi_{l-1} \circ \cdots \circ \psi_{0} \circ \psi_{-1}\right)(u),
$$

where the number $l$ (which depends on $u$ ) is given by the formula

$$
l=h-2+m
$$

where $h$ is the height of $u$ and $m$ is the maximum number of consecutive valleys of $\left(\psi_{h-2} \circ \psi_{h-3} \circ \cdots \circ \psi_{0} \circ \psi_{-1}\right)(u)$ at height $h-1$ and $\psi_{-1}$ denotes the identity mapping.

Since each $\psi_{i}$ is an involution, we can easily check that $\psi$ is a bijection with

$$
\psi^{-1}(u)=\left(\psi_{-1} \circ \psi_{0} \circ \psi_{1} \circ \cdots \circ \psi_{l^{\prime}}\right)(u)
$$


where

$$
l^{\prime}= \begin{cases}h-1, & \text { if } u \text { contains at least one valley at height } h-1 ; \\ h-2, & \text { otherwise. }\end{cases}
$$

Finally, since by Lemma $5.4 \psi(u), \psi_{i}(u)$ contain the same number of valleys at height $i$ and $\psi(u)$ does not contain valleys at any height greater than $h-2$, for $h \geq 2$ we have

$$
\hat{p}(u)=\sum_{i=0}^{h-2} p_{i+2}(u)=\sum_{i=0}^{h-2} v_{i}\left(\psi_{i}(u)\right)=v(\psi(u)),
$$

whereas for $h=1$ we have $\hat{p}(u)=0=v(\psi(u))$.

In Fig. 4 the main steps of the generation of $\psi(u)$ are exhibited for a particular $u$.
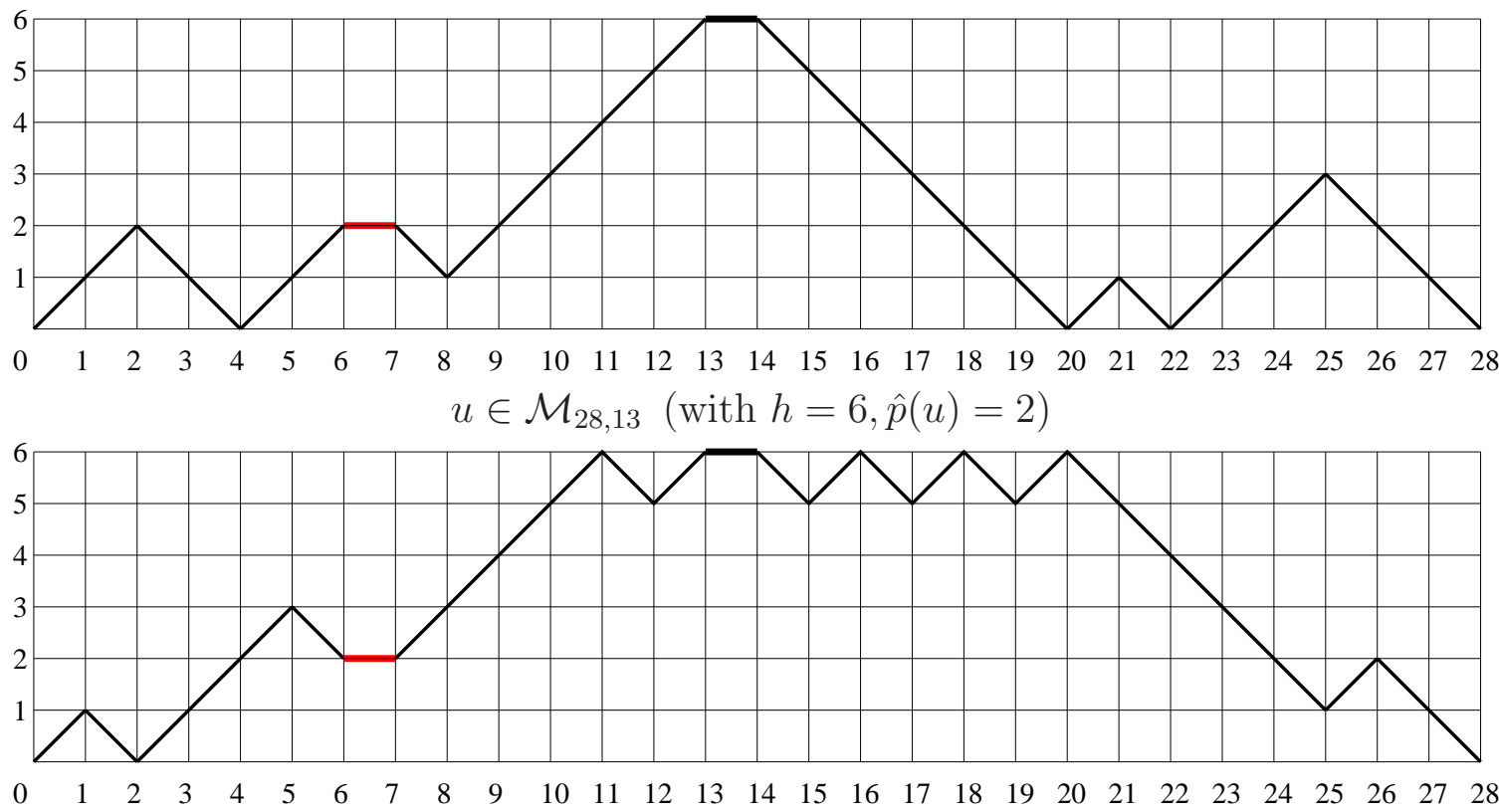

$\left(\psi_{4} \circ \psi_{3} \circ \psi_{2} \circ \psi_{1} \circ \psi_{0} \circ \psi_{-1}\right)(u)($ with $m=3)$

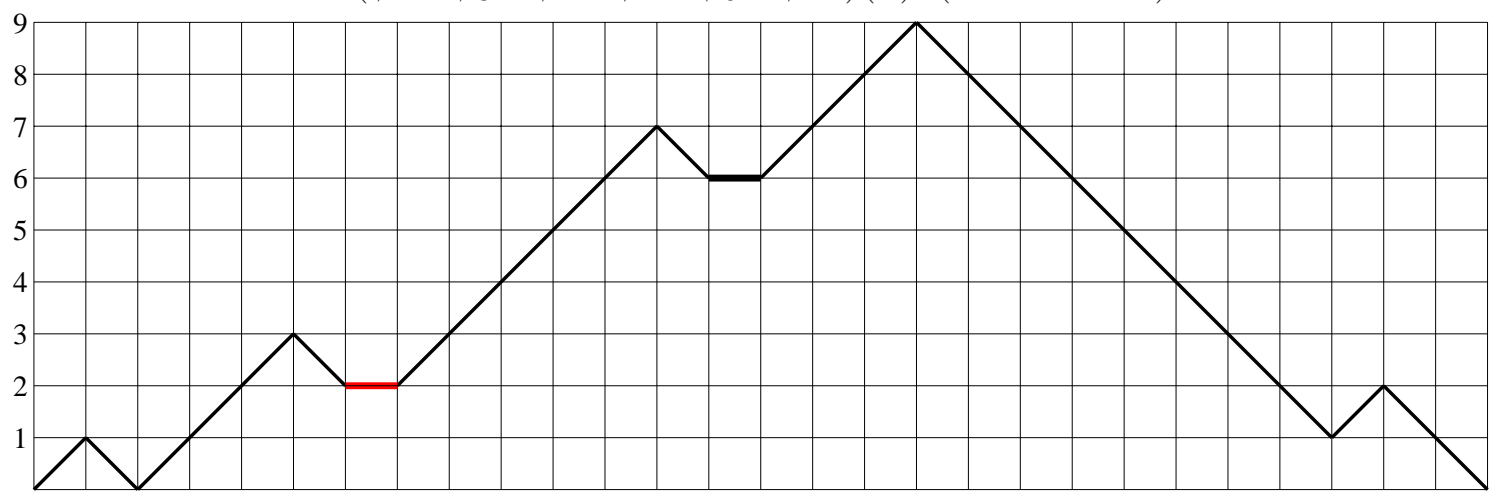

$\begin{array}{lllllllllllllllllllllllllllll}0 & 1 & 2 & 3 & 4 & 5 & 6 & 7 & 8 & 9 & 10 & 11 & 12 & 13 & 14 & 15 & 16 & 17 & 18 & 19 & 20 & 21 & 22 & 23 & 24 & 25 & 26 & 27 & 28\end{array}$ $\psi(u)=\left(\psi_{7} \circ \psi_{6} \circ \psi_{5} \circ \psi_{4} \circ \psi_{3} \circ \psi_{2} \circ \psi_{1} \circ \psi_{0} \circ \psi_{-1}\right)(u)\left(\right.$ with $\left.h=9, l^{\prime}=7, v(\psi(u))=2\right)$

Figure 4: The generation of $\psi(u)$ 


\section{Acknowledgement}

The authors would like to thank the anonymous referee for his detailed and constructive suggestions on the original manuscript.

\section{References}

[1] L. Alonso, Uniform generation of a Motzkin word, Theoret. Comput. Sci., 134 (1994), $529-536$.

[2] E. Barcucci, A. Del Lungo, E. Pergola and R. Pinzani, A construction for enumerating $k$-coloured Motzkin paths, Proc. of the First Annual International Conference on Computing and Combinatorics (Ding-Zhu Du and Ming Li, eds.) Springer, 1995, pp. 254-263.

[3] D. Callan, Two bijections for Dyck path parameters, Preprint, (2004), 4pp. http://www.arxiv.org/abs/math.CO/0406381.

[4] A. de Médicis and X. G. Viennot, Moments des q-Polynômes de Laguerre et la bijection de Foata-Zeilberger, Adv. in Appl. Math. 15 (1994) 262-304.

[5] E. Deutsch, A bijection on Dyck paths and its consequences, Discrete Math. 179 (1998), 253-256 (Corrigendum: 187 (1998), 297).

[6] E. Deutsch, An involution on Dyck paths and its consequences, Discrete Math. 204 (1999), 163-166.

[7] E. Deutsch, Dyck path enumeration, Discrete Math. 204 (1999), 167-202.

[8] E. Deutsch and L. W. Shapiro, A bijection between ordered trees and 2-Motzkin paths and its many consequences, Discrete Math. 256 (2002), 655-670.

[9] R. Donaghey and L. W. Shapiro, Motzkin numbers, J. Combin. Theory Ser. A 23 (1977), 291-301.

[10] H. W. Gould, Combinatorial Identities, Morgantown, 1972.

[11] F. Harary and R. C. Read, The enumeration of tree-like polyhexes, Proc. Edinb. Math. Soc. 17 (1970), 1-13.

[12] T. Mansour, Counting peaks at height $k$ in a Dyck path, J. Integer Seq. 5 (2002), Article 02.1.1.

[13] P. Peart and W.-J. Woan, Dyck paths with no peaks at height $k$, J. Integer Seq. 4 (2001), Article 01.1.3.

[14] A. Panayotopoulos and A. Sapounakis, On the prime decomposition of Dyck words, J. Combin. Math. Combin. Comput. 40 (2002), 33-39.

[15] A. Panayotopoulos and A. Sapounakis, On Motzkin words and noncrossing partitions, Ars Combin. 69 (2003), 109-116.

[16] J. Riordan, Combinatorial Identities, John Wiley and Sons, 1968.

[17] A. Sapounakis and P. Tsikouras, On k-colored Motzkin words, J. Integer Seq. 7 (2004), Article 04.2.5. 
[18] N. J. A. Sloane, (2005), The On-Line Encyclopedia of Integer Sequences, published electronically at http://www.research.att.com/njas/sequences.

[19] R. P. Stanley, Enumerative Combinatorics, Vol. 2, Cambridge University Press, 1999.

[20] R. A. Sulanke, Bijective recurrences for Motzkin paths, Adv. in Appl. Math. 27 (2001), 627-640. 